\title{
MERS-CoV in sheep, goats, and cattle, United Arab Emirates, 2019: Virological and serological investigations reveal an accidental spillover from dromedaries
}

Pia Weidinger ${ }^{1}$, Jolanta Kolodziejek ${ }^{1}$, Jeremy Camp ${ }^{1}$, tom loney ${ }^{2}$, Dafalla Ahmed ${ }^{3}$, Sathishkumar Ramaswamy ${ }^{4}$, Ahmad Abou Tayoun ${ }^{2}$, Victor Max Corman ${ }^{5}$, and Norbert Nowotny ${ }^{1}$

${ }^{1}$ Veterinarmedizinische Universitat Wien

${ }^{2}$ Mohammed Bin Rashid University of Medicine and Health Sciences College of Medicine ${ }^{3} \mathrm{Al}$ Ain City Municipality

${ }^{4}$ Al Jalila Children's Specialty Hospital

${ }^{5}$ Charite Universitatsmedizin Berlin

July 21, 2021

\begin{abstract}
The recent COVID-19 pandemic has demonstrated again the global threat posed by emerging zoonotic coronaviruses. During the past two decades alone, humans have experienced the emergence of several coronaviruses, such as SARS-CoV in 2003, MERS-CoV in 2012, and SARS-CoV-2 in 2019. To date, MERS-CoV has been detected in 27 countries, with a case fatality ratio of approximately $34.5 \%$. Similar to other coronaviruses, MERS-CoV presumably originated from bats; however, the main reservoir and primary source of human infections are dromedary camels. Other species within the Camelidae family, such as Bactrian camels, alpacas, and llamas, seem to be susceptible to the infection as well, although to a lesser extent. In contrast, susceptibility studies on sheep, goats, cattle, pigs, chickens, and horses obtained divergent results. In the present study, we tested nasal swabs and/or sera from 55 sheep, 45 goats, and 52 cattle, collected at the largest livestock market in the United Arab Emirates, where dromedaries are also traded, for the presence of MERS-CoV nucleic acid by RT-qPCR, and for specific antibodies by immunofluorescence assay (IFA). All sera were negative for MERS-CoV-reactive antibodies, but the nasal swab of one sheep $(1.8 \%$ ) was positive for MERS-CoV nucleic acid. Next generation sequencing (NGS) of the complete $N$ gene of the sheep-derived MERS-CoV revealed >99\% nucleotide identity to MERS-CoV sequences of five dromedaries in nearby pens and to three reference sequences. The NGS sequence of the sheep-derived MERS-CoV was confirmed by conventional RT-PCR of a part of the $N$ gene and subsequent Sanger sequencing. All MERS-CoV sequences clustered within clade B, lineage 5. In conclusion, our study shows that non-camelid livestock, such as sheep, goats, and cattle do not play a major role in MERS-CoV epidemiology. The one sheep that tested positive most likely reflects an accidental viral spillover event from infected dromedaries in nearby pens.
\end{abstract}

MERS-CoV in sheep, goats, and cattle, United Arab Emirates, 2019: Virological and serological investigations reveal an accidental spillover from dromedaries

Short running title (less than 70 characters):

Study on MERS-CoV in sheep, goats, and cattle, UAE, 2019

Pia Weidinger ${ }^{1}$, Jolanta Kolodziejek ${ }^{1}$, Jeremy V. Camp ${ }^{1,2}$, Tom Loney ${ }^{3}$, Dafalla O. Kannan ${ }^{4}$, Sathishkumar Ramaswamy $^{5}$, Ahmad Abou Tayoun ${ }^{3,5}$, Victor M. Corman ${ }^{6}$, Norbert Nowotny ${ }^{1,3}$ 
${ }^{1}$ Viral Zoonoses, Emerging and Vector-Borne Infections Group, Institute of Virology, University of Veterinary Medicine, Vienna, Austria

${ }^{2}$ Center for Virology, Medical University of Vienna, Austria

${ }^{3}$ College of Medicine, Mohammed Bin Rashid University of Medicine and Health Sciences, Dubai, United Arab Emirates

${ }^{4} \mathrm{Al}$ Ain City Municipality, Al Ain, United Arab Emirates

${ }^{5}$ Al Jalila Genomics Center, Al Jalila Children's Specialty Hospital, Dubai, United Arab Emirates

${ }^{6}$ Institute of Virology, Charité-Universitätsmedizin Berlin, Humboldt-Universität zu Berlin, Berlin Institute of Health, and German Centre for Infection Research (DZIF), Partner Site Charité, Berlin, Germany

*Corresponding author: Norbert Nowotny, Viral Zoonoses, Emerging and Vector-Borne Infections Group, Institute of Virology, University of Veterinary Medicine Vienna, Veterinaerplatz 1, 1210 Vienna, Austria. Phone: +431250772403; Fax: +431250772390; Email: Norbert.Nowotny@vetmeduni.ac.at

ORCID IDs

Pia Weidinger https://orcid.org/0000-0003-4028-4151

Jolanta Kolodziejek https://orcid.org/0000-0001-5736-3644

Jeremy V. Camp https://orcid.org/0000-0002-9040-5786

Tom Loney https://orcid.org/0000-0003-1687-6587

Dafalla O. Kannan https://orcid.org/0000-0001-5819-0028

Sathishkumar Ramaswamy https://orcid.org/0000-0002-7467-6658

Ahmad Abou Tayoun https://orcid.org/0000-0002-9134-1673

Victor M. Corman https://orcid.org/0000-0002-3605-0136

Norbert Nowotny https://orcid.org/0000-0002-3548-571X

\section{Ethics Statement}

The authors confirm that the ethical policies of the journal, as noted on the journal's author guidelines page, have been adhered to. No ethical approval was required for sampling of the involved animals.

\section{Summary}

The recent COVID-19 pandemic has demonstrated again the global threat posed by emerging zoonotic coronaviruses. During the past two decades alone, humans have experienced the emergence of several coronaviruses, such as SARS-CoV in 2003, MERS-CoV in 2012, and SARS-CoV-2 in 2019. To date, MERS-CoV has been detected in 27 countries, with a case fatality ratio of approximately $34.5 \%$. Similar to other coronaviruses, MERS-CoV presumably originated from bats; however, the main reservoir and primary source of human infections are dromedary camels. Other species within the Camelidae family, such as Bactrian camels, alpacas, and llamas, seem to be susceptible to the infection as well, although to a lesser extent. In contrast, susceptibility studies on sheep, goats, cattle, pigs, chickens, and horses obtained divergent results. In the present study, we tested nasal swabs and/or sera from 55 sheep, 45 goats, and 52 cattle, collected at the largest livestock market in the United Arab Emirates, where dromedaries are also traded, for the presence of MERS-CoV nucleic acid by RT-qPCR, and for specific antibodies by immunofluorescence assay (IFA). All sera were negative for MERS-CoV-reactive antibodies, but the nasal swab of one sheep (1.8 \%) was positive for MERS-CoV nucleic acid. Next generation sequencing (NGS) of the complete $N$ gene of the sheep-derived MERS-CoV revealed $>99 \%$ nucleotide identity to MERS-CoV sequences of five dromedaries in nearby pens and to three reference sequences. The NGS sequence of the sheep-derived MERS-CoV was confirmed by 
conventional RT-PCR of a part of the $N$ gene and subsequent Sanger sequencing. All MERS-CoV sequences clustered within clade B, lineage 5 . In conclusion, our study shows that non-camelid livestock, such as sheep, goats, and cattle do not play a major role in MERS-CoV epidemiology. The one sheep that tested positive most likely reflects an accidental viral spillover event from infected dromedaries in nearby pens.

\section{Keywords}

Sheep, goat, cattle, Middle East respiratory syndrome, Middle East respiratory syndrome coronavirus, MERS-CoV

\section{Introduction}

Since the 1930s, members of the family Coronaviridae have been identified in humans and many different animal species, such as cats, dogs, horses, pigs, cattle, mice, and fowl . In the last 20 years, three zoonotic coronaviruses have emerged into the human population:Severe acute respiratory syndrome-related coronavirus (SARS-CoV) in 2003, Middle East respiratory syndrome-related coronavirus(MERS-CoV) in 2012, and most recently, SARS-CoV-2 in 2019, which led to the so far most devastating pandemic of the $21^{\text {st }}$ century . Since 2012, MERS-CoV has been detected in 27 countries, with $84.0 \%$ of cases found in Saudi Arabia, and a case fatality ratio of approximately $34.5 \%$ (WHO, 2019). Similar to other coronaviruses, MERS-CoV presumably originated from bats; however, the main reservoir and primary source of human infections are dromedaries (Camelus dromedarius ). Although dromedaries usually remain asymptomatic, or develop only

mild symptoms due to MERS-CoV infection, it has been shown that they can shed considerable amount of virus . Studies on Bactrian camels (Camelus bactrianus), hybrid camels, alpacas (Vicugna pacos ), llamas (Lama glama), and even pigs (Sus scrofa), have revealed that these animals are also susceptible to MERSCoV infection (Adney et al., 2019; Lau et al., 2020; Reusken et al., 2016; Vergara-Alert et al., 2017). In contrast, studies on sheep, goats, cattle, horses, and chickens have indicated that, although sheep and goats may produce antibodies to MERS-CoV, none of these species effectively shed the virus (Adney et al., 2016; Hemida et al., 2013; Reusken, Ababneh et al., 2013; Vergara-Alert et al., 2017). A study about domestic mammals in contact with infected dromedaries found MERS-CoV nucleic acid in nasal swabs not only from sheep and goats, but also from a cow and donkeys (Kandeil et al., 2019). To provide more information about the potential exposure and infection of other livestock, we performed a survey for MERS-CoV at a livestock market in the United Arab Emirates (UAE), as live animal markets are known to be common sources of virus spillover.

\section{Materials and Methods}

\section{Sampling}

Sampling was performed at the largest national livestock market in the UAE, the Al Ain central market in the emirate of Abu Dhabi, in October 2019. The market is divided into three main areas, with mostly mixed sheep and goat pens located between camel and cattle stalls (Fig. 1). The distance between camel pens and the closest pens holding sheep and goats is approximately $20 \mathrm{~m}$.

In total, we sampled 152 animals, which appeared healthy at the time of sampling. Nasal swabs and blood samples were taken from 55 sheep (41 female and 14 male) with an age range from three months to four years (mean of 19 months), and 45 goats (31 female and 14 male) from three to 40 months old (mean of 12 months). In addition, we collected blood samples from 52 cattle (43 female and nine male) from one to six years (mean of 3.8 years). Nasal swabs were collected in tubes containing a virus inactivation solution (DNA/RNA Shield, ZymoResearch, Irvine, CA, USA). Serum was separated from each blood sample by centrifugation. All samples were then stored at $-80 \mathrm{degC}$ at the laboratory of the College of Medicine, Mohammed Bin Rashid University of Medicine and Health Sciences, Dubai, UAE, before shipment on dry ice to the University of Veterinary Medicine, Vienna, Austria, for virological and serological investigations.

\section{MERS-CoV nucleic acid screening}

Nasal swabs of sheep $(n=55)$ and goats $(n=45)$, as well as serum samples of all animals $(n=152)$ were screened 
for MERS-CoV nucleic acid by reverse transcription real-time (RT-q)PCR. For this, $200 \mu$ of each sample were subjected to automated nucleic acid extraction employing QIAamp Viral RNA Mini Kit on QIAcube HT (both Qiagen, Hilden, Germany). MERS-CoV-specific RT-qPCR was performed using qScript XLT One Step RT-qPCR ToughMix (QuantaBio, MA, USA) and previously described primers and probe in the open reading frame $(O R F) 1 a$ gene . RT-qPCR-positive samples were additionally tested by conventional RTPCRs (OneStep RT-PCR Kit, Qiagen, Hilden, Germany) employing two previously published primer pairs within the nucleocapsid $(N)$ and ORF1b genomic regions of MERS-CoV. Amplicons from the RT-PCRs were subjected to Sanger sequencing (Eurofins Genomics, Ebersberg, Germany). In addition, next generation sequencing (NGS) was performed on putative positive samples and five selected MERS-CoV-positive samples from dromedaries in nearby pens. For this, adapter sequences were trimmed using bcl2fastq2 conversion software v2.20.0, and high-quality sequencing reads were aligned to three reference MERS-CoV genome sequences (GenBank acc. numbers KF192507, MG757604 and MK462253) using BWA v0.7.17. Consensus sequences were generated for the $N$ gene using GATK v3.8-1-0 and BCFtools v1.3.1. Multiple sequence alignment of the $N$ gene of MERS-CoV $(1.2 \mathrm{~kb})$ derived from one sheep and five dromedary camels was performed using MAFFT v7.455.

\section{MERS-CoV antibody testing}

Altogether 148 serum samples from sheep $(n=55)$, goats $(n=45)$, and cattle $(n=48)$ were available in sufficient volumes to perform MERS-CoV antibody testing. MERS-CoV spike protein-based immunofluorescence assay (IFA) was done as described previously. Initial screening was performed with sample dilutions of 1:40, retesting of putative positive (questionable) samples was done with a dilution series of 1:10 - 1:320. Detection was facilitated using 1:200 dilutions of donkey-anti sheep, donkey-anti goat, or goat-anti bovine secondary antibodies, all labelled with Alexa Fluor 488 (Dianova, Hamburg, Germany).

\section{Results}

\section{MERS-CoV nucleic acid and antibody prevalence}

The nasal swab of one sheep (1.8\%) repeatedly tested positive for MERS-CoV nucleic acid by RT-qPCR (Ct values 32.5-34.8; Table 1), and the nasal swab of one goat tested borderline positive (Ct values 38.139.4; Table 1). The positive sheep was a two-year-old female, presumably locally raised Nuaimi breed. It was kept in a pen at the market used for sheep only, which was the second closest pen to the camel area (approximately $25 \mathrm{~m}$ away; Fig. 1, Supplementary Table 1). The borderline positive goat, a one-year-old locally raised male Omani goat, was kept in one of the pens directly adjacent to the camel pens, with only approximately $20 \mathrm{~m}$ between them (Fig. 1, Supplementary Table 1).

The RT-qPCR-positive sheep sample was confirmed by two different RT-PCRs. The obtained sequences [lengths without primer sequences: $N-293$ bp (GenBank acc. number: MZ558082), ORF1b - 341 bp (GenBank acc. number: MZ558083)] were $>99 \%$ identical to many MERS-CoV sequences belonging to clade B, lineage 5 [according to the classification by, including human and camel strains from the UAE, Saudi Arabia, South Korea, Jordan, Qatar, and the United Kingdom. Unfortunately, we were not able to uncover the complete MERS-CoV genomes using NGS; however we succeeded to establish the complete $N$ gene sequences of the virus-positive sheep and five selected MERS-CoV-positive dromedaries (Figure 2). The sheep-derived $N$ gene sequences obtained by NGS and Sanger sequencing were identical. By NGS, the average sequencing coverage of the $N$ gene from the sheep (S90_N) and five camel samples (C53_N, C5_N, C6_N, C28_$\mathrm{N}$, and $\mathrm{C} 50 \_\mathrm{N}$ ) was $>6 \mathrm{X}$ across all samples (Figure $2 \mathrm{~A}$ ). The percentage of sequence identity of the $N$ gene of the same samples along with three reference MERS-CoV sequences (GenBank acc. numbers KF192507, MG757604 and MK462253) revealed that the sheep sample showed high nucleotide identity (>99\%) with all other MERS-CoV sequences (Figure 2B). In detail, multiple sequence alignment revealed five positions of nucleotide variations between the sheep-derived MERS-CoV sequence and the camel-derived sequences and/or reference sequences (representative positions 533 and 637 of the $N$ gene are shown in Figure 2C). The GenBank accession numbers of the complete MERS-CoV $N$ gene sequences are: sheep-derived (S90_N): MZ558076; dromedary-derived: C53_N - MZ558080; C5_N - MZ558077; C6_N - MZ558078; C28_N - 
MZ558079; and C50_N - MZ558081. Unfortunately, the borderline-positive result of the goat sample could not be confirmed by either RT-PCR or NGS.

The serological survey revealed that, although in the initial screening with 1:40 dilutions 15 serum samples (eight sheep, four goats, three cattle) appeared questionable, the presence of antibodies could not be confirmed in any sample by retesting using a dilution series of 1:10 - 1:320 (Supplementary Tables 1 and 2).

\section{Discussion}

Previous studies have demonstrated that, besides dromedaries, several other members of the Camelidae family are susceptible to MERS-CoV infection, such as Bactrian camels, alpacas, and llamas, although human contact infections have only been reported from dromedary camels . However, the existing data on the susceptibility of non-camelids are conflicting. Our results are in line with a series of publications demonstrating that non-camelids do not play a major role in the transmission cycle . For instance, in a seroepidemiological study in Saudi Arabia (2010-2013), no antibodies were detected in sheep, goats, cattle, or chickens, but a high prevalence was found in camels . Similarly, after an experimental challenge of sheep, goats, and horses by intranasal MERS-CoV inoculation, none of the animals effectively shed the virus, and neutralizing antibodies were only found in goats . Further regarding the family Equidae, including horses, donkeys, and mules, although viral replication has been observed in primary horse kidney cells, no antibodies have been found in any of 1,053 equid sera collected in the UAE and Spain . A study on livestock cell lines from the Arabian Peninsula showed that MERS-CoV can replicate in goat, alpaca, and dromedary cells, in contrast to sheep, cattle, rodent, and insectivore cells . In a study experimentally challenging animals from France and Spain, MERS-CoV nucleic acid in the respiratory tract as well as seroconversion were detected only in llamas and pigs, but not in sheep and horses . A serosurvey in a MERS-CoV-affected area in Jordan identified MERS-CoV neutralising antibodies in the sera of all camels but none in goats and cattle, while $4.8 \%$ of sheep sera reacted with MERS-CoV antigen. In a monitoring study of several mammalian species in direct contact with infected dromedaries in Egypt, Tunisia, and Senegal (2015-2017), antibodies were identified in an astonishing $55.6 \%$ of sheep from Senegal, $1.8 \%$ of sheep from Tunisia, and $0.9 \%$ of goats from Egypt. MERS-CoV RNA was detected in nasal swabs from $1.2 \%$ sheep and $4.1 \%$ goats from Egypt and Senegal, as well as $1.9 \%$ cattle and $7.1 \%$ donkeys from Egypt. In conclusion, it seems that several non-camelid species may become infected with MERS-CoV during viral spillover events when they are in direct contact with virus-shedding dromedaries. Maybe this susceptibility is due to the close relatedness of the Betacoronaviruses (specifically their receptors) infecting camels and especially bovids, but also humans, porcines, and equids. However, whether these animals are implicated in the MERS-CoV transmission cycle remains unknown, but seems to be highly unlikely.

The fact that none of the sheep, goats, and cattle in our survey tested positive for antibodies indicates that none of them had previously experienced a MERS-CoV infection, although with an age range of three months to six years, all animals could have encountered the virus in the past. Moreover, as the average time of stay at the market for goats is usually 7-10 days, 10-14 days for sheep, and between 14 and 30 days for cattle, there is also a not negligible risk of infection at the market. However, the nasal swab of one sheep $(1.8 \%)$ contained MERS-CoV nucleic acid (Supplementary Table 1), suggesting either a current infection, or a spillover event from nearby dromedaries, which appears to be more likely since no antibodies were detected and the sheep was kept in a pen in close proximity to the camel pens (approximately $25 \mathrm{~m}$ away; Fig. 1). Of note, we additionally sampled 90 dromedaries at the same market and at the same time as the non-camelids, of which $35.6 \%$ were positive for MERS-CoV nucleic acid, and another $24.4 \%$ tested borderline positive (Ct values 38.6-41.2). Moreover, $91.1 \%$ of the 90 camel sera were MERS-CoV antibody-positive by ELISA, and $96.7 \%$ by IFA (data can be provided upon request), further supporting the high prevalence of MERS-CoV infections in dromedaries. This hypothesis is also supported by our latest study, in which we screened 76 dromedaries during spring and autumn 2019 at the same market, and found MERS-CoV nucleic acid in $57.9 \%$ of their nasal swabs .

The positive sheep from this study was a two-year-old female, presumably locally raised Nuaimi breed. However, due to the fact that sex was not equally distributed across the animals we sampled (115 females, 
$75.7 \%$, compared to 37 males, $24.3 \%$ ), no conclusions can be drawn regarding the susceptibility of the different sexes. The same is true for the different breeds, with only one positive animal, we cannot infer any breed-specific genetic predispositions to MERS-CoV infection.

In conclusion, our study shows that non-camelids such as sheep, goats, and cattle do not play a major role in MERS-CoV epidemiology. The one sheep that tested RNA-positive in our study most likely reflects a viral spillover event from infected dromedaries in nearby pens. Furthermore, serology revealed that the sheep did not develop an antibody response, suggesting that the infection was very recent, or the sheep only carried viral particles on its nasal mucosa without actually being infected.

\section{Acknowledgements}

The authors thank the General Manager of Al Ain City Municipality, H.E. Dr. Matar Mohammed Saif $\mathrm{Al}$ Nuaimi, and his team, including Dr. Babiker Mohammed Osman, for supporting the study, as well as Hassan Mohammed Alkaabi, Head of Veterinary Services, Hashim Ahmed Saeed and Md. Helal Ahmed for assistance with sampling at the livestock market. We are grateful for the help of Noushad Karuvantevida, Athiq Ahmed Wahab, and Abubakkar Babuhan, from the Mohammed Bin Rashid University of Medicine and Health Sciences, in facilitating the study.

This work was supported by research grants of the College of Medicine, Mohammed Bin Rashid University of Medicine and Health Sciences, Dubai, United Arab Emirates (grant no. MBRU-CM-RG2018-14 to N.N., and grant no. MBRU-CM-RG2019-13 to T.L.).

\section{Conflict of Interest Statement}

The authors declare no conflict of interest.

\section{References}

Adney, D. R., Brown, V. R., Porter, S. M., Bielefeldt-Ohmann, H., Hartwig, A. E., \& Bowen, R. A. (2016). Inoculation of goats, sheep, and horses with MERS-CoV does not result in productive viral shedding. Viruses ,8 (8). https://doi.org/10.3390/v8080230

Adney, D. R., Letko, M., Ragan, I. K., Scott, D., van Doremalen, N., Bowen, R. A., \& Munster, V. J. (2019). Bactrian camels shed large quantities of Middle East respiratory syndrome coronavirus (MERS-CoV) after experimental infection. Emerging Microbes 83 Infections ,8 (1), 717-723. https://doi.org/10.1080/22221751.2019.1618687

Adney, D. R., van Doremalen, N., Brown, V. R., Bushmaker, T., Scott, D., Wit, E. de, . . . Munster, V. J. (2014). Replication and shedding of MERS-CoV in upper respiratory tract of inoculated dromedary camels.Emerging Infectious Diseases , 20 (12), 1999-2005. https://doi.org/10.3201/eid2012.141280

Azhar, E. I., El-Kafrawy, S. A., Farraj, S. A., Hassan, A. M., Al-Saeed, M. S., Hashem, A. M., \& Madani, T. A. (2014). Evidence for camel-to-human transmission of MERS coronavirus. The New England Journal of Medicine , 370 (26), 2499-2505. https://doi.org/10.1056/NEJMoa1401505

Corman, V. M., Müller, M. A., Costabel, U., Timm, J., Binger, T., Meyer, B., . . Drosten, C. (2012). Assays for laboratory confirmation of novel human coronavirus (hCoV-EMC) infections. Eurosurveillance ,17 (49), 20334. https://doi.org/10.2807/ese.17.49.20334-en

Corman, V. M., Eckerle, I., Bleicker, T., Zaki, A., Landt, O., Eschbach-Bludau, M., . . Drosten, C. (2012). Detection of a novel human coronavirus by real-time reverse-transcription polymerase chain reaction. Eu- 
ro Surveillance : Bulletin Europeen Sur Les Maladies Transmissibles = European Communicable Disease Bulletin , 17 (39). https://doi.org/10.2807/ese.17.39.20285-en

Corman, V. M., Ithete, N. L., Richards, L. R., Schoeman, M. C., Preiser, W., Drosten, C., \& Drexler, J. F. (2014). Rooting the phylogenetic tree of Middle East respiratory syndrome coronavirus by characterization of a conspecific virus from an African bat. Journal of Virology ,88 (19), 11297-11303. https://doi.org/10.1128/JVI.01498-14

Drosten, C., Günther, S., Preiser, W., van der Werf, S., Brodt, H.-R., Becker, S., . . . Doerr, H. W. (2003). Identification of a novel coronavirus in patients with severe acute respiratory syndrome. The New England Journal of Medicine , 348 (20), 1967-1976. https://doi.org/10.1056/NEJMoa030747

Eckerle, I., Corman, V. M., Müller, M. A., Lenk, M., Ulrich, R. G., \& Drosten, C. (2014). Replicative capacity of MERS coronavirus in livestock cell lines. Emerging Infectious Diseases , 20 (2), 276-279. https://doi.org/10.3201/eid2002.131182

Farag, E. A. B. A., Reusken, C. B. E. M., Haagmans, B. L., Mohran, K. A., Raj, V. S., Pas, S. D., . . . Koopmans, M. P. G. (2015). High proportion of MERS-CoV shedding dromedaries at slaughterhouse with a potential epidemiological link to human cases, Qatar 2014. Infection Ecology 83 Epidemiology , 5 (1), 28305. https://doi.org/10.3402/iee.v5.28305

Hemida, M. G., Perera, R. A., Wang, P., Alhammadi, M. A., Siu, L. Y., Li, M., . . Peiris, M. (2013). Middle East Respiratory Syndrome (MERS) coronavirus seroprevalence in domestic livestock in Saudi Arabia, 2010 to 2013. Euro Surveillance : Bulletin Europeen Sur Les Maladies Transmissibles = European Communicable Disease Bulletin , 18 (50), 20659. https://doi.org/10.2807/1560-7917.es2013.18.50.20659

Hemida, M. G., Chu, D. K. W., Poon, L. L. M., Perera, R. A. P. M., Alhammadi, M. A., Ng, H.-Y., . . . Peiris, M. (2014). Mers coronavirus in dromedary camel herd, Saudi Arabia. Emerging Infectious Diseases ,20 (7), 1231-1234. https://doi.org/10.3201/eid2007.140571

Hemida, M. G., Chu, D. K. W., Chor, Y. Y., Cheng, S. M. S., Poon, L. L. M., Alnaeem, A., \& Peiris, M. (2020). Phylogenetic analysis of MERS-CoV in a camel abattoir, Saudi Arabia, 2016-2018. Emerging Infectious Diseases , 26 (12), 3089-3091. https://doi.org/10.3201/eid2612.191094

Kandeil, A., Gomaa, M., Shehata, M., El-Taweel, A., Kayed, A. E., Abiadh, A., . . Kayali, G. (2019). Middle East respiratory syndrome coronavirus infection in non-camelid domestic mammals. Emerging Microbes $\mathscr{B}$ Infections , 8 (1), 103-108. https://doi.org/10.1080/22221751.2018.1560235

Lado, S., Elbers, J. P., Plasil, M., Loney, T., Weidinger, P., Camp, J. V., . . Burger, P. A. (2021). Innate and adaptive immune genes associated with MERS-CoV infection in dromedaries. Cells , 2021,10 (6), 1291. https://doi.org/10.3390/cells10061291

Lai, M. M.C., \& Cavanagh, D. (1997). The molecular biology of coronaviruses. In K. Maramorosch, F. A. Murphy, \& A. J. Shatkin (Eds.), Advances in virus research (Vol. 48, pp. 1-100). Academic Press. https://doi.org/10.1016/S0065-3527(08)60286-9

Lau, S. K. P., Li, K. S. M., Luk, H. K. H., He, Z., Teng, J. L. L., Yuen, K.-Y., . . Woo, P. C. Y. (2020). Middle East respiratory syndrome coronavirus antibodies in Bactrian and hybrid camels from Dubai.MSphere , 5 (1). https://doi.org/10.1128/mSphere.00898-19

Meyer, B., García-Bocanegra, I., Wernery, U., Wernery, R., Sieberg, A., Müller, M. A., . . Eckerle, I. (2015). Serologic assessment of possibility for MERS-CoV infection in equids. Emerging Infectious Diseases ,21 (1), 181-182. https://doi.org/10.3201/eid2101.141342

Muhairi, S. A., Hosani, F. A., Eltahir, Y. M., Mulla, M. A., Yusof, M. F., Serhan, W. S., . . Abdelazim, A. S. (2016). Epidemiological investigation of Middle East respiratory syndrome coronavirus in dromedary camel farms linked with human infection in Abu Dhabi Emirate, United Arab Emirates. Virus Genes , 52 (6), 848-854. https://doi.org/10.1007/s11262-016-1367-1 
Nowotny, N., \& Kolodziejek, J. (2014). Middle East respiratory syndrome coronavirus (MERS-CoV) in dromedary camels, Oman, 2013. Euro Surveillance : Bulletin Europeen Sur Les Maladies Transmissibles = European Communicable Disease Bulletin , 19 (16), 20781. https://doi.org/10.2807/1560-7917.es2014.19.16.20781

Reusken, C. B., Ababneh, M., Raj, V. S., Meyer, B., Eljarah, A., Abutarbush, S., . . Koopmans, M. P. (2013). Middle East respiratory syndrome coronavirus (MERS-CoV) serology in major livestock species in an affected region in Jordan, June to September 2013. Advance online publication. https://doi.org/10.2807/15607917.es2013.18.50.20662

Reusken, C. B., Schilp, C., Raj, V. S., Bruin, E. de, Kohl, R. H., Farag, E. A., . . Koopmans, M. P. (2016). MERS-CoV infection of alpaca in a region where MERS-CoV is endemic. Emerg Infect Dis , 22 (6), 1129-1131. https://doi.org/10.3201/eid2206.152113

Reusken, C. B., Haagmans, B. L., Müller, M. A., Gutierrez, C., Godeke, G.-J., Meyer, B., . . . Koopmans, M. P. G. (2013). Middle East respiratory syndrome coronavirus neutralising serum antibodies in dromedary camels: a comparative serological study. The Lancet Infectious Diseases ,13 (10), 859-866. https://doi.org/10.1016/S1473-3099(13)70164-6

Su, S., Wong, G., Shi, W., Liu, J., Lai, A. C.K., Zhou, J., . . . Gao, G. F. (2016). Epidemiology, genetic recombination, and pathogenesis of coronaviruses. Trends in Microbiology , 24 (6). https://doi.org/10.1016/j.tim.2016.03.003

Van Doremalen, N., Miazgowicz, K. L., Milne-Price, S., Bushmaker, T., Robertson, S., Scott, D., . . Munster, V. J. (2014). Host species restriction of Middle East respiratory syndrome coronavirus through its receptor, dipeptidyl peptidase 4. Journal of Virology ,88 (16), 9220-9232. https://doi.org/10.1128/JVI.00676-14

Vergara-Alert, J., van den Brand, J. M. A., Widagdo, W., Muñoz, M., Raj, S., Schipper, D., . . Segalés, J. (2017). Livestock susceptibility to infection with Middle East respiratory syndrome coronavirus. Emerging Infectious Diseases , 23 (2), 232-240. https://doi.org/10.3201/eid2302.161239

WHO (2019). MERS global summary and assessment of risk . Retrieved from World Health Organization website: https://apps.who.int/iris/bitstream/handle/10665/326126/WHO-MERS-RA-19.1-eng.pdf

Wölfel, R., Corman, V. M., Guggemos, W., Seilmaier, M., Zange, S., Müller, M. A., . . . Wendtner, C. (2020). Virological assessment of hospitalized patients with COVID-2019. Nature , 581 (7809), 465-469. https://doi.org/10.1038/s41586-020-2196-x

Wu, A., Peng, Y., Huang, B., Ding, X., Wang, X., Niu, P., . . Jiang, T. (2020). Genome composition and divergence of the novel coronavirus (2019-nCoV) originating in China. Cell Host \& Microbe ,27 (3), 325-328. https://doi.org/10.1016/j.chom.2020.02.001

Zaki, A. M., van Boheemen, S., Bestebroer, T. M., Osterhaus, A. D. M. E., \& Fouchier, R. A. M. (2012). Isolation of a novel coronavirus from a man with pneumonia in Saudi Arabia. The New England Journal of Medicine , 367 (19), 1814-1820. https://doi.org/10.1056/NEJMoa1211721

Table 1. Results of the MERS-CoV screening in sheep, goats, and cattle. Nasal swabs from sheep and goats, and sera from all animals were tested for MERS-CoV nucleic acid by ORF1a RT-qPCR. Serum samples from all but four animals (i.e. cattle sera with insufficient volumes) were investigated for MERS-CoV-specific antibodies by IFA.

\begin{tabular}{llllll}
\hline Species & $\mathbf{n ~ ( f e m a l e / m a l e )}$ & Age range (average) & Nucleic acid-positive & Nucleic acid-positive & Antibody \\
& & & Nasal swabs & Serum samples & \\
Sheep & $55(41 / 14)$ & $3-48$ months (19 months) & $1(1.8 \%)$ & 0 & 0 \\
Goat & $45(31 / 14)$ & $3-40$ months (12 months) & 1 borderline $(2.2 \%)$ & 0 & 0 \\
Cattle & $52(43 / 9)$ & $1-6$ years (3.8 years) & 0 & 0 & 0 \\
\hline
\end{tabular}


Figure 1: Image of the Al Ain central market in Abu Dhabi. Camel pens are located on the left side, cattle stalls on the right side, and sheep and goats are kept in between. The distance between camel pens and the closest pens holding sheep and goats is approximately $20 \mathrm{~m}$ (separated by a road). The red asterisk indicates sheep pen S18, in which the MERS-CoV-positive sheep was sampled, the green asterisk indicates the mixed pen S17, in which the borderline positive goat was kept. Image retrieved from Google Maps.

Figure 2: Next generation sequencing analysis of the MERS-CoVN gene of sheep and camels. A) Sequencing coverage of the MERS-CoV $N$ gene derived from one sheep (S90_N) and five camels (C53_N, C5_N, C6_N, C28_N, and C50_N). The average coverage of the $N$ gene across all samples was $>6 \mathrm{X}$. X axis $=$ $N$ gene of MERS-CoV $(1.2 \mathrm{~kb})$ and $\mathrm{Y}$ axis = sequencing coverage; red line = sheep-derived MERS-CoV, blue lines = dromedary-derived MERS-CoV. B) Percentage of $N$ gene sequence identity of the same samples along with three reference MERS-CoV sequences (GenBank acc. numbers KF192507, MG757604 and MK462253). The sample collected from the sheep showed high nucleotide identity $(>99 \%)$ with all camel-derived MERS$\mathrm{CoV}$ sequences as well as the reference sequences. C) Multiple sequence alignment of the $N$ gene of the same MERS-CoV samples $(1.2 \mathrm{~kb})$. Two short representative regions with nucleotide sequence variations are shown: positions 533 and 637 of the $N$ gene (highlighted by a red vertical box); conserved positions are indicated by asterisks $\left(^{*}\right)$. Multiple sequence alignment was performed using MAFFT v7.455.

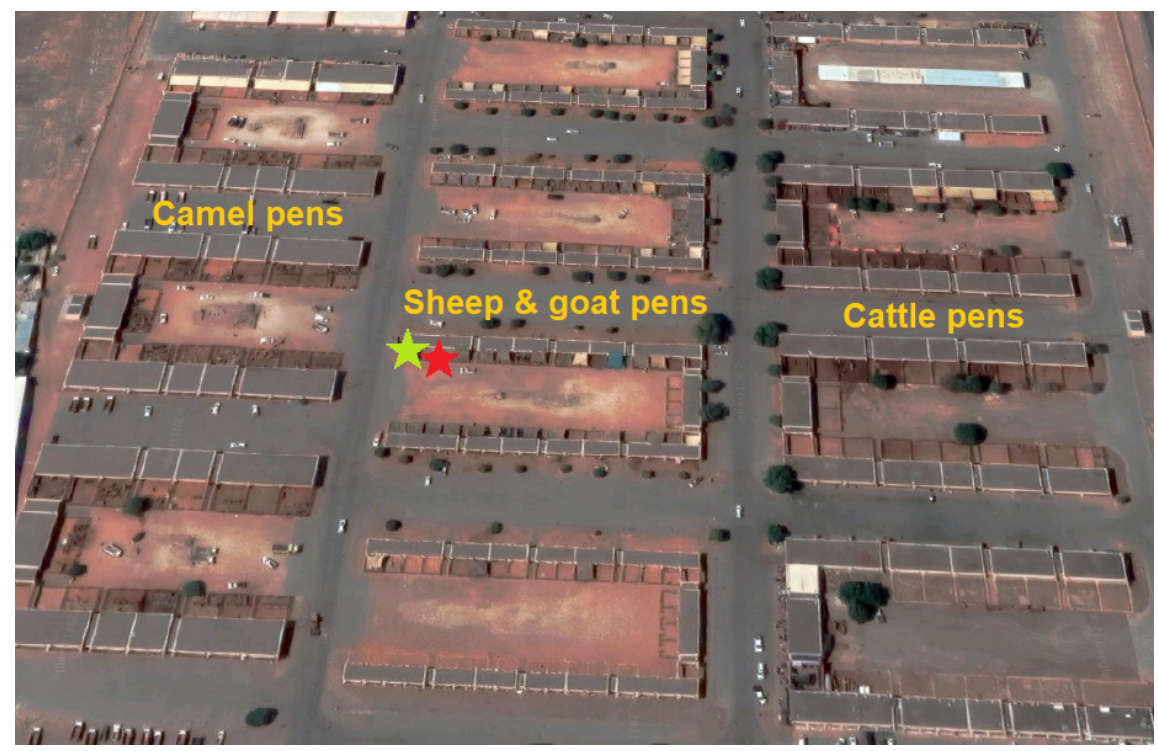


a)

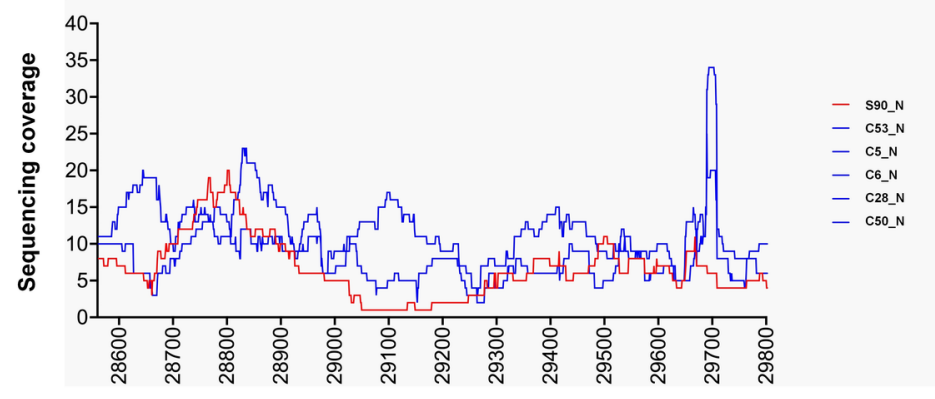

$N$ gene of MERS-CoV (1.2 kb)

b)

\begin{tabular}{|c|c|c|c|c|c|c|c|c|c|}
\hline & $\begin{array}{l}\text { KF192507_N } \\
\text { (UAE 2013) }\end{array}$ & $\begin{array}{c}\text { MG757604_N } \\
\text { (KSA 2016) }\end{array}$ & $\begin{array}{c}\text { MK462253_N } \\
\text { (KSA 2018) }\end{array}$ & S90_N & C53_N & C5_N & $\mathrm{C} 6 \_\mathrm{N}$ & C28_N & $\mathrm{C} 50 \_\mathrm{N}$ \\
\hline KF192507_N (UAE 2013) & 100 & 99.84 & 99.60 & 99.84 & 99.60 & 99.60 & 99.60 & 99.76 & 99.76 \\
\hline MG757604_N (KSA 2016) & 99.84 & 100 & 99.43 & 99.67 & 99.43 & 99.43 & 99.43 & 99.59 & 99.59 \\
\hline MK462253_N (KSA 2018) & 99.60 & 99.43 & 100 & 99.60 & 99.84 & 99.84 & 99.84 & 99.84 & 99.68 \\
\hline Sheep S90_N & 99.84 & 99.67 & 99.60 & 100 & 99.60 & 99.76 & 99.76 & 99.76 & 99.92 \\
\hline Camel C53_N & 99.60 & 99.43 & 99.84 & 99.60 & 100 & 99.84 & 99.84 & 99.84 & 99.68 \\
\hline Camel C5_N & 99.60 & 99.43 & 99.84 & 99.76 & 99.84 & 100 & 100 & 99.84 & 99.84 \\
\hline Camel C6_N & 99.60 & 99.43 & 99.84 & 99.76 & 99.84 & 100 & 100 & 99.84 & 99.84 \\
\hline Camel C28_N & 99.76 & 99.59 & 99.84 & 99.76 & 99.84 & 99.84 & 99.84 & 100 & 99.84 \\
\hline Camel C50_N & 99.76 & 99.59 & 99.68 & 99.92 & 99.68 & 99.84 & 99.84 & 99.84 & 100 \\
\hline
\end{tabular}

c)

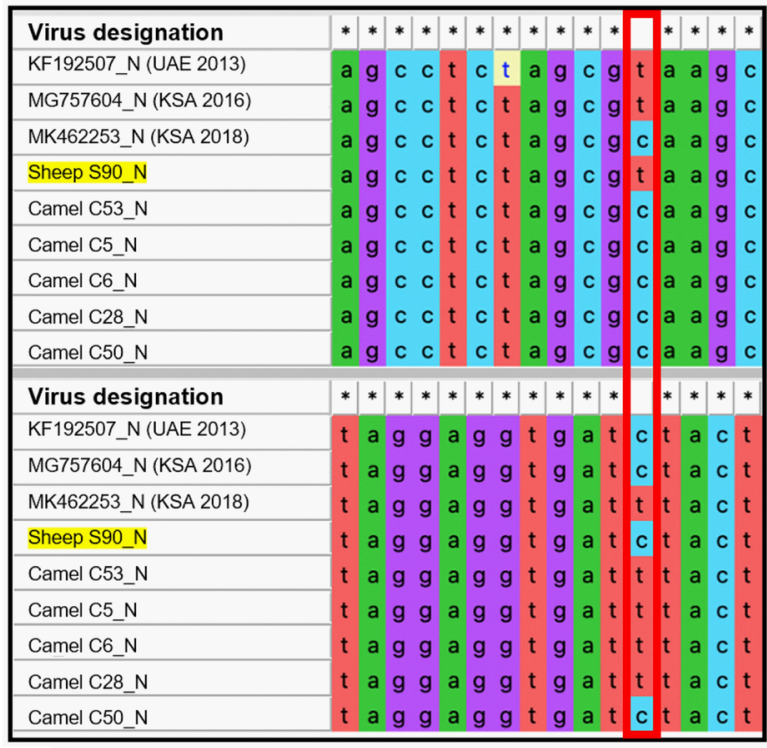

